\title{
Immer kürzer, immer schneller?
}

\section{DRG-System, Verkürzung der Liegezeiten im Krankenhaus und Folgen für die Patienten und die Behandlungsabläufe}

\begin{abstract}
Eine der weitreichendsten gesundheitspolitischen Strukturreformen der letzten Jahre ist die Einführung der diagnosebezogenen Fallpauschalen (DRGs) als neues Entgeltsystem im Krankenhaus. Zuvor wurde überwiegend nach tagesgleichen Pflegesätzen und Sonderentgelten und seit 1996 bei rund $25 \%$ überwiegend chirurgischen Leistungen bereits nach Fallpauschalen abgerechnet. Vereinfacht gesagt werden im DRG-System alle Patienten je nach Diagnose, Prozedur und Schweregrad in bestimmte Fallgruppen eingeordnet, für die das Krankenhaus eine pauschale Vergütung erhält. Dadurch ist die Abrechnung des Einzelfalls unabhängig von der Dauer des Aufenthaltes und den tatsächlich für das Krankenhaus entstandenen Kosten.
\end{abstract}

\section{Bernard Braun, Petra Buhr, Rolf Müller}

Nach den Vorstellungen des Gesetzgebers sollen hierdurch einerseits bestimmte Fehlanreize des alten Vergütungssystems beseitigt und die Wirtschaftlichkeit der Leistungserbringung in den Krankenhäusern erhöht werden, wodurch die Ausgaben im Gesundheitssystem sinken sollen. Andererseits soll die Patientenversorgung durch den gleichzeitigen Ausbau von Maßnahmen zur Qualitätssicherung verbessert werden (Deutscher Bundestag 2001: 26). Viele Beobachter ${ }^{1}$ gehen allerdings davon aus, dass die DRG-Einführung auch oder sogar eher negative Folgen für die Patientenversorgung haben wird. Befürchtet werden beispielsweise selektive Patientenaufnahmen, Entlassungen in instabilem gesundheitlichen Zustand, Vorenthaltung medizinisch notwendiger Leistungen sowie insgesamt eine geringere Patientenorientierung und geringere Ganzheitlichkeit der Behandlung.

Um die Auswirkungen der DRG-Einführung möglichst prozessnah untersuchen und negative Folgen nötigenfalls verhindern zu können, hat der Gesetzgeber ein Kontrollinstrument vorgeschrieben und ein Implementationsprinzip vorgeschlagen: Im $\S 17 \mathrm{~b}$ (8) KHG ist eine wissenschaftliche Begleitforschung vorgeschrieben, deren Ergebnisse erstmals 2005 veröffentlicht werden sollten. Hinzu kommt ein neuartiges Verständnis politischer Regulierung als langjährigem Veränderungsprozess in einem „lernenden System“. Um eine möglichst große Praxisnähe zu gewährleisten übertrug der Gesetzgeber einen Großteil der Anpassungsarbeiten an Einrichtungen der gemeinsamen Selbstverwaltung von Krankenkassen und Krankenhäusern (vor allem das Institut für das Ent-

Dr. Bernard Braun, Dr. Petra Buhr, Dr. Rolf Müller (alle Zentrum für Sozialpolitik der Universität Bremen, Forschungseinheit Gesundheitspolitik und Evaluation medizinischer Versorgung) geltsystem im Krankenhaus (InEK)). In der Zeit vom optionalen Einstieg ins DRG-System bis zur vollkommenen Implementierung der DRG-basierten Abrechungen ist also die mehrfache Überprüfung und Veränderung der Versorgungs- und Organisationsbedingungen nicht Ausdruck von lästigen Pannen, sondern gewollt. Trotz der klaren gesetzlichen Vorschriften wurden jedoch bisher offiziell keine Forschungsprojekte ausgeschrieben. Die meisten Stellen und Forschergruppen, die sich mit den Folgen der DRG-Einführung beschäftigen, untersuchen jeweils nur Teilaspekte der DRG-Einführung. ${ }^{2}$ Das einzige Projekt, das einen umfassenden Ansatz hat, ist das Projekt „Wandel von Medizin und Pflege im DRGSystem (WAMP)“, das seit 2002 vom Zentrum für Sozialpolitik der Universität Bremen und dem Wissenschaftszentrum Berlin für Sozialforschung durchgeführt wird. ${ }^{3}$ In dem Projekt werden die Folgen der DRG-Einführung mit einem Design untersucht, das auf mehrmaligen repräsentativen standardisierten Befragungen von Ärzten, Pflegekräften und Patienten, kontinuierlichen Auswertungen von Routinedaten einer Krankenkasse sowie wiederholten Fallstudien in Krankenhäusern aufbaut. ${ }^{4}$ Es handelt sich also um ein multidimensionales Längsschnittdesign. Wichtig für die Durchführung valider Veränderungsmessungen ist, dass die erste Welle der standardisierten Befragungen vor bzw. kurz nach der verpflichtenden Einführung der DRGs stattfand und Vergleichsdaten für die Beurteilung der weiteren Entwicklung existieren. ${ }^{5}$ Die zweite Welle der Patienten- und Ärztebefragung fand Ende 2005 bzw. Anfang 2006 statt; die zweite Pflegekräftebefragung ist im September 2006 ins Feld gegangen. Die dritte standardisierte Befragungswelle ist für 2007 geplant. Die erste Welle der Interviews mit allen relevanten Akteuren in den für Fallstudien ausgesuchten Krankenhäusern fand im Winter 2004/05 statt. Die zweite Befragung ist ebenfalls für 2007 geplant. Zu dem Zeitpunkt dürfte der wirtschaftliche Druck auf die 
Krankenhäuser wegen der schrittweisen Anpassung der krankenhausindividuellen Basisfallwerte an einen einheitlichen landesweiten Basisfallwert größer geworden sein. ${ }^{6}$ WAMP stellt somit Informationen bereit, die in dieser Form und in diesem Umfang bisher nicht erhoben worden sind und auch nicht mehr erhoben werden können.

Dieser Beitrag konzentriert sich auf einen Aspekt, der besonders häufig in einem Atemzug mit der DRG-Einführung genannt wird, nämlich die Verkürzung der Liegezeiten. Um die damit verknüpften Hoffnungen (z. B. Ausgabensenkung und Verbesserung der Versorgungsqualität) und Befürchtungen (z. B. zu frühe, „blutige“ Entlassungen) kreist ein Großteil der versorgungspolitischen DRG-Debatte der letzten Jahre. Es wird zunächst gefragt, ob sich die Liegezeiten tatsächlich verändern, wie die Patienten diese Entwicklung bewerten und ob es Hinweise auf zu frühe Entlassungen gibt. Im zweiten Schritt wird untersucht, ob die Behandlungsstrukturen und -prozesse in deutschen Krankenhäusern geeignet sind, Behandlungszeiten ohne Qualitätsverlust zu reduzieren. Dazu werden der Stand des Entlassungs- und Überleitungsmanagements, das Ausmaß der Standardisierung von Behandlungsprozessen (z. B. durch Clinical Pathways oder Leitlinien) sowie die Qualität der Kooperation zwischen Ärzten und Pflegekräften betrachtet. Sofern nichts anderes vermerkt, handelt es sich im Folgenden immer um Ergebnisse der verschiedenen Erhebungen aus dem Projekt WAMP.

\section{Zu frühe Entlassungen?}

Die durchschnittliche Verweildauer im Krankenhaus geht seit Jahren zurück. Dauerte eine vollstationäre Behandlung 1991 noch durchschnittlich 14 Tage, lag die Dauer 2004 nur noch bei 8,7 Tagen (Statistisches Bun- desamt 2006). Ein besonderer DRG-Effekt, also eine sprunghafte Abnahme der Liegezeiten nach 2002, ist bisher nicht zu erkennen. Seit 1998 sinkt die durchschnittliche Verweildauer um ca. 0,3 Liegetage pro Jahr. $\mathrm{Zu}$ berücksichtigen ist allerdings auch, dass die durchschnittliche Fallschwere, der so genannte Case-MixIndex (CMI), von 2002 bis $2004 \mathrm{um}+10,7 \%$ (AOK Patienten) angestiegen ist (Friedrich/Günster 2006: 168). Dies kann mehrere Gründe haben: Die Kodierung der Krankenhausfälle könnte ausführlicher vorgenommen worden sein, es könnte sich um eine Tendenz des Upgradings der DRGs handeln, um in jedem Fall auf der wirtschaftlich sicheren Seite zu sein, oder immer kränkere Menschen werden in immer kürzerer Zeit behandelt.

Kürzere Liegezeiten müssen nicht per se negativ sein. Im Gegenteil: Es gibt Indizien dafür, dass die Liegezeiten in der Vergangenheit häufig unnötig lang waren und u.a. lange Liegezeiten zu unerwünschten Nebeneffekten wie z. B. Krankenhausinfektionen führen. Im Vorfeld der DRG-Einführung machte jedoch das Schlagwort von der „blutigen“ Entlassung die Runde. Hiermit sollte die Befürchtung ausgedrückt werden, Patienten könnten zu einem Zeitpunkt entlassen oder in Rehabilitationseinrichtungen verlegt werden, $\mathrm{zu}$ dem sie eigentlich noch nicht entlassungsfähig sind. Ein mögliches Indiz für zu frühe Entlassungen sind Wiederaufnahmen mit der gleichen Diagnose nach der Entlassung aus dem Krankenhaus. Soweit ersichtlich hat die Rehospitalisierungsquote seit Einführung der DRGs jedoch nicht zugenommen.

Auch der Großteil der Patienten hat weder 2002 noch 2005 mit der Dauer seines Krankenhausaufenthaltes ein Problem. Etwa $70 \%$ halten den Entlasszeitpunkt für genau richtig, für knapp jeden Zehnten war der Aufenthalt zu kurz. Bei einigen Krankheitsbildern sieht das Bild jedoch anders aus: Bei Krebserkrankungen und rheumati-

Graphik 1: Anteil der Patienten mit ausgewählten Aufnahmegründen, die meinten, ihr Krankenhausaufenthalt „hätte etwas länger sein müssen", 2002 und 2005

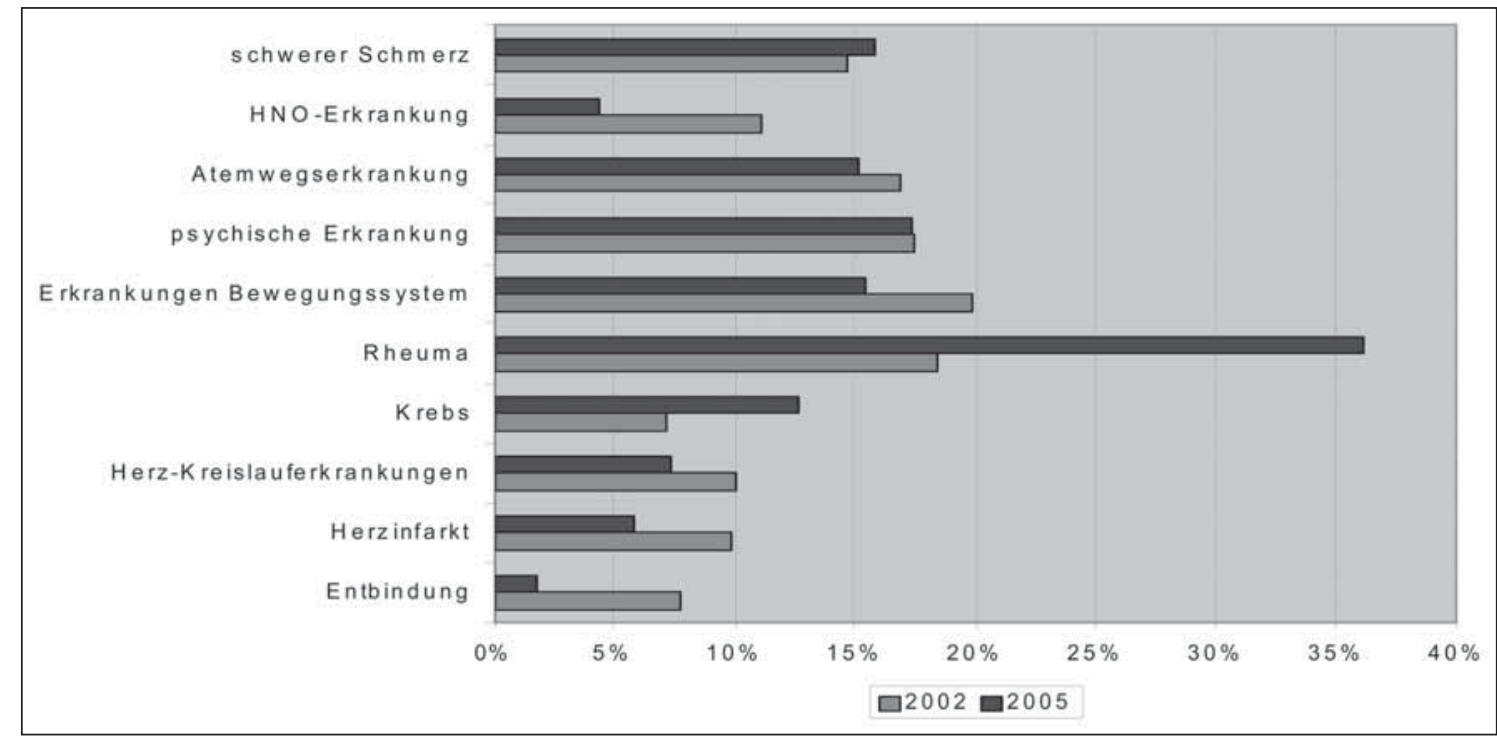

Quelle: Zusammenstellung aus Ergebnissen des Projektes WAMP 
schen Beschwerden hat sich der Anteil der Patienten, die den Krankenhausaufenthalt für zu kurz halten, seit 2002 verdoppelt. Im Jahr 2005 meint sogar mehr als jeder dritte Rheumapatient, der Krankenhausaufenthalt hätte etwas länger sein müssen. Auch der Krankenhaustyp spielt eine Rolle dafür, wie die Dauer des Aufenthaltes bewertet wird: Bei vielen Krankheitsbildern, z. B. Krebs und Erkrankungen des Bewegungsapparates, sagen Patienten, die in privaten Krankenhäusern behandelt wurden, häufiger als Patienten in öffentlichen und freigemeinnützigen Häusern, der Entlassungszeitpunkt sei zu früh gewesen. Bei Rheuma fallen dagegen eher die öffentlichen Krankenhäuser aus dem Rahmen.

Die Dauer des Krankenhausaufenthaltes deckt sich also nicht immer mit den Patientenbedürfnissen. Und auch nach Ansicht eines Fünftels der befragten Ärzte erfolgt die Entlassung häufig oder durchgängig zu früh.

\section{Reibungsverluste beim Übergang in den nachstatio- nären Bereich?}

Auch wenn „blutige“ Entlassungen bisher nicht im großen Stil praktiziert werden: Die kürzeren Liegezeiten bringen es mit sich, dass die Patienten zu einem Zeitpunkt entlassen werden, zu dem sie noch nicht wieder im Vollbesitz ihrer Kräfte sind und kontinuierliche Behandlung und Unterstützung außerhalb des Krankenhauses benötigen. Damit kommt der Vorbereitung der Entlassung und der Überleitung in den nachstationären Bereich eine noch deutlich höhere Bedeutung zu als schon bisher (vgl. Wingenfeld 2005). Wenn die Patienten nicht über das angemessene Verhalten nach der Entlassung aufgeklärt werden und die Fortsetzung der Behandlung zwischen Krankenhausärzten und niedergelassenen Ärzten nicht abgestimmt wird, besteht die Gefahr, dass sich die Genesung verzögert bzw. Komplikationen auftreten oder nicht rechtzeitig erkannt werden.
Der Anteil der Patienten, die keine der fünf von uns abgefragten Entlassungsleistungen erhielten, ist zwar, mit Ausnahme der Patienten der privaten Krankenhäuser, zwischen 2002 und 2005 zurückgegangen. Von einer befriedigenden Situation kann gleichwohl nicht die Rede sein: Bedenklich ist, dass auch 2005 mehr als 10\% der Patienten keine verständliche Aufklärung über den Sinn und Zweck der Medikamenteneinnahme nach der Entlassung erhielten, 15\% keine Hinweise erhielten, welche gesundheitlichen Warnsignale sie beachten müssen, mit mehr als $20 \%$ nicht besprochen wurde, wann sie wieder Alltagsaktivitäten aufnehmen können, etwa 25\% keine Erklärung darüber erhielten, wie sie sich selbst bei der Genesung helfen können und in fast $60 \%$ der Fälle die Angehörigen nicht einbezogen wurden - obwohl sie all diese Leistungen für notwendig erachteten.

Die kürzeren Liegezeiten haben also bisher nicht zu einer deutlichen Verbesserung der Entlassungsvorbereitung geführt. Vielmehr wird ein Teil der Patienten nach kurzer Liegezeit in mehrfacher Hinsicht unvorbereitet aus dem Krankenhaus entlassen.

Auch die für eine nahtlose Weiterversorgung von Patienten wichtige Kooperation des Krankenhauses mit den niedergelassenen Ärzten, ambulanten und stationären Pflegediensten und Rehabilitationseinrichtungen ist verbesserungsbedürftig. Der Anteil der Ärzte, die angaben, Kooperationen des Krankenhauses mit ambulanten Fachärzten oder Einrichtungen der Rehabilitation, ambulanten oder stationären Pflege existierten entweder gar nicht oder stünden nur auf dem Papier, stieg zwischen 2004 und 2006 sogar durchweg an.

Der Übergang in den nachstationären Bereich funktioniert also nicht so reibungslos wie es bei kürzer werdenden Liegezeiten notwendig ist. Ein Teil der Patienten erhält keinerlei Entlassvorbereitung und nur eine Minderheit kann mit einer koordinierten Weiterbehandlung im ambulanten Bereich rechnen.

Graphik 2: Anteil der Patienten, der die jeweilige Entlassungsleistung nicht erhielt, 2002 und 2005

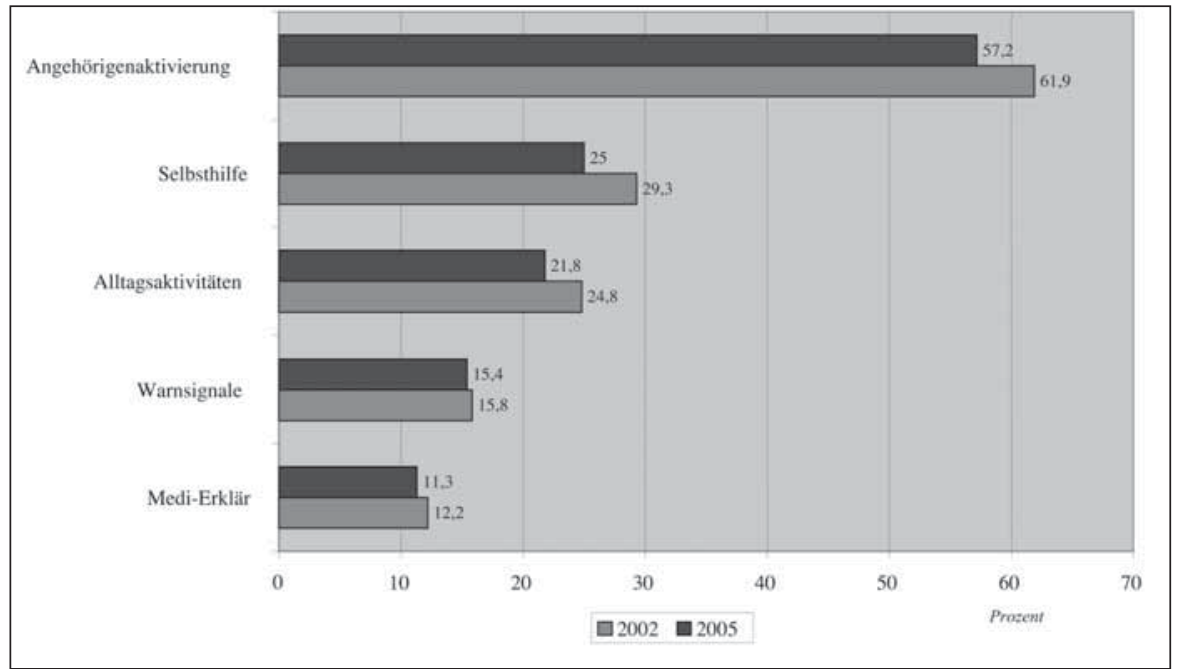

Quelle: Zusammenstellung aus Ergebnissen des Projektes WAMP 
Graphik 3: Anteil der Ärzte, die die ausgewählten Kooperationen als nicht oder nur auf dem Papier existent bezeichneten, 2004 und 2006

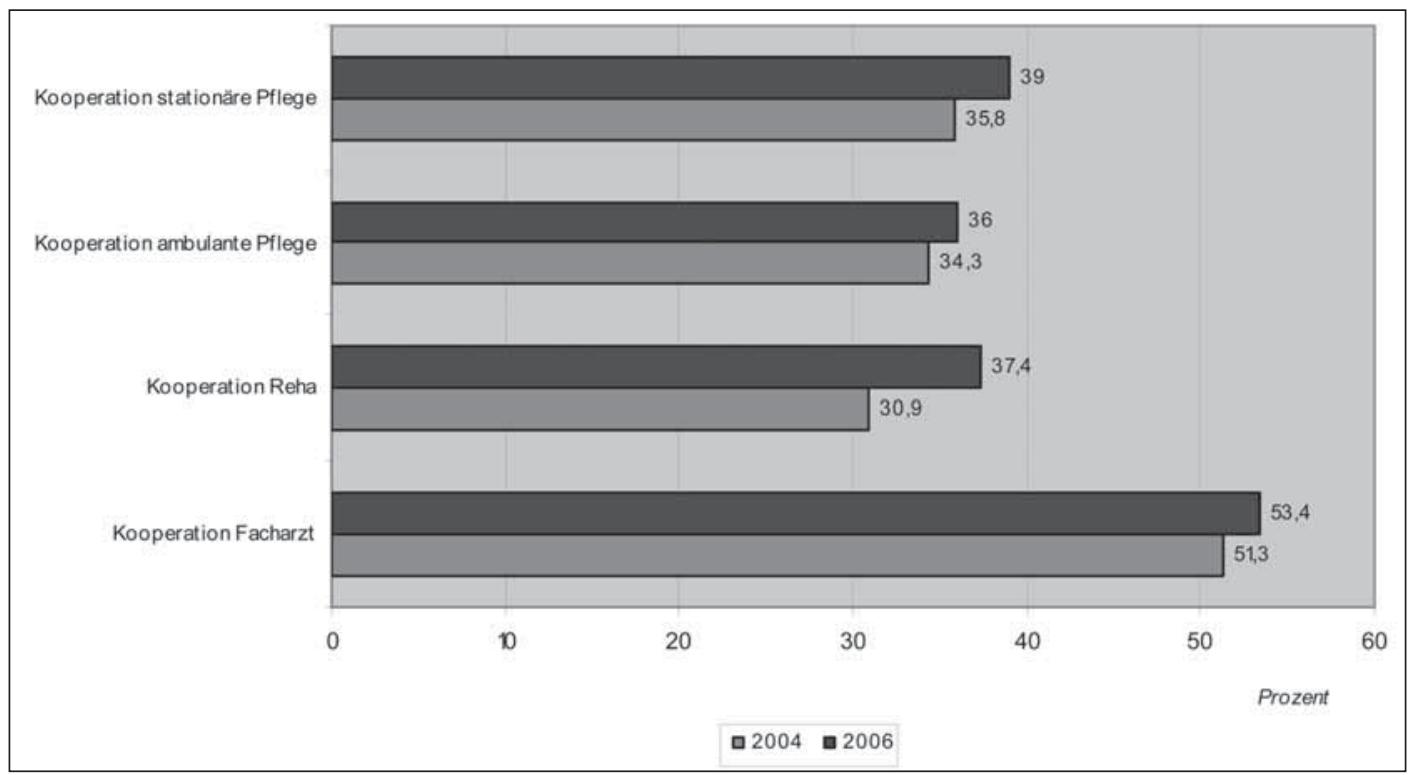

Quelle: Zusammenstellung aus Ergebnissen des Projektes WAMP

\section{Reibungsverluste bei der Aufnahme der Patienten?}

Aber nicht nur das Entlassmanagement, auch der Aufnahmevorgang ist wichtig, wenn Behandlungserfolge in immer kürzerer Zeit erzielt werden sollen. Ein Indikator für die Qualität des Aufnahmemanagements ist der Informationsstand der Ärzte bei der Aufnahme des Patienten ins Krankenhaus. Wenn die Ärzte über den Gesundheitszustand des Patienten und vorangegangene Behandlungen vollständig informiert sind, können Untersuchungen und Arbeitsabläufe besser geplant, Umwege, Fehleinschätzungen, Doppeluntersuchungen und weitere Verunsicherungen von sowieso verunsicherten Patienten vermieden werden. Kurzum: Ein optimales Aufnahmemanagement kann dazu beitragen, die Patienten zügiger und problemgerechter zu behandeln und letztlich auch schneller entlassen zu können.

Etwa jeder 10. Patient gibt an, die Ärzte im Krankenhaus hätten beim Erstkontakt nicht alle notwendigen Informationen gehabt. Nimmt man die Patienten hinzu, die nicht wussten, wie der Informationsstand ihrer Ärzte aussah, war die Aufnahmesituation für 15 bis $20 \%$ aller eingewiesenen Patienten durch Informationsmängel

Graphik 4: Informationsstand der Ärzte - aus Sicht der Patienten - über den Gesundheitszustand und die Vorbehandlung 2002 und 2005

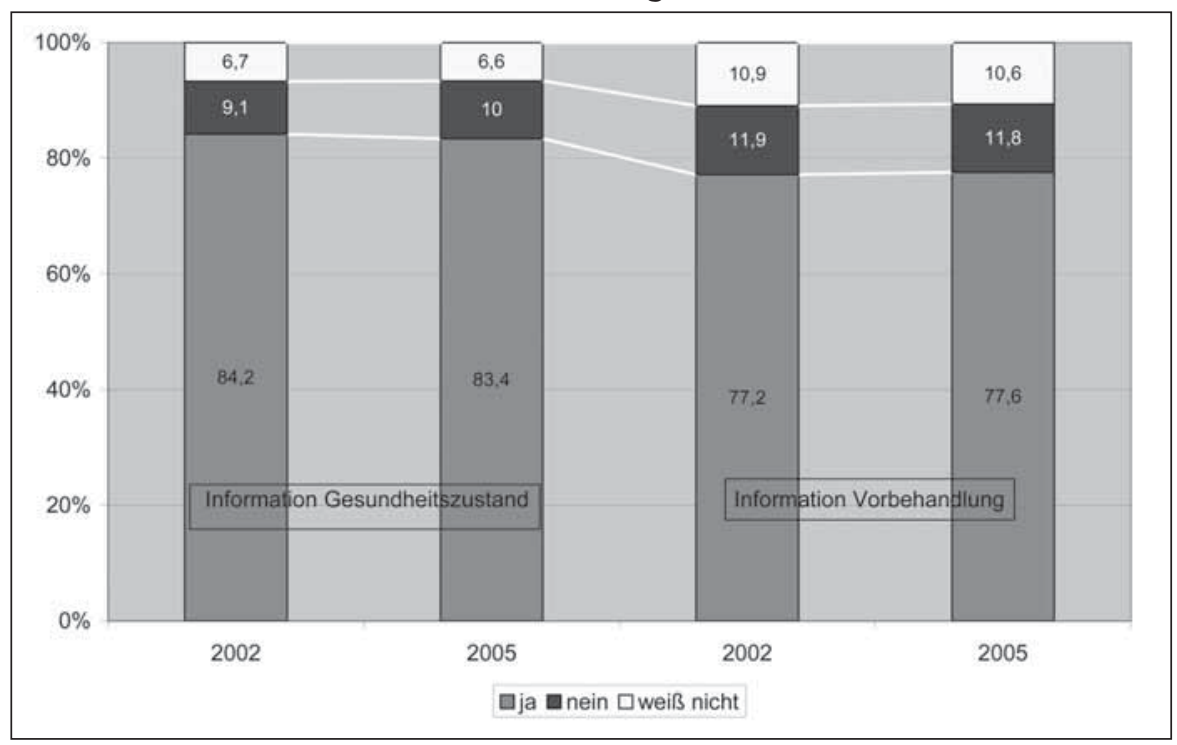

Quelle: Zusammenstellung aus Ergebnissen des Projektes WAMP 
ihrer Ärzte getrübt. Schwerwiegend und unerwartet ist die Beobachtung, dass zwischen 2002 und 2005 aus Patientensicht keine Besserung der Situation eingetreten ist.

Die Wahrnehmung der Patienten wird durch die Erfahrungen der befragten Krankenhaus-Ärzte bestätigt. Diese sehen die Situation sogar noch etwas kritischer: Auf die Frage, ob ihnen bei der Aufnahme „sämtliche Unterlagen über den Gesundheitszustand und die Vorbehandlung zur Verfügung" ständen, sagten $44 \%$, dies wäre häufig oder sogar meistens der Fall, während 56\% angaben, ihnen läge beim Erstkontakt nur selten bis nie eine vollständige Patientenakte vor. Auch aus Sicht der Ärzte hat sich nach eineinhalb Jahren DRG-Einführung nicht viel verändert: Bei der zweiten Ärztebefragung lag sogar etwas mehr von ihnen, nämlich knapp $57 \%$ nur selten oder nie eine vollständige Patientenakte vor.

\section{Reibungsverluste im Verlauf der Behandlung?}

Wenn die meisten Patienten nur noch wenige Tage im Krankenhaus verbringen, müssen Untersuchungen und Behandlungen in immer kürzerer Zeit ablaufen. Im Vorfeld der DRG-Einführung wurde deshalb auch die Notwendigkeit herausgestellt, Prozesse und Behandlungsabläufe stärker zu optimieren, zu koordinieren und zu standardisieren (vgl. Bähr-Heintze 2004; Dietrich et al. 2004; Loch 2005; Oberender 2005; Wingenfeld 2005). Hierdurch sollen Zeit und Kosten gespart (indem zum Beispiel auf Doppeluntersuchungen verzichtet wird) und zugleich Qualität gesichert werden.

Während sich an den Schnittstellen vom und zum ambulanten Bereich wie oben gezeigt kaum Verbesserun- gen ergeben haben, sind die Prozesse innerhalb des Krankenhauses in den letzten Jahren offenbar stärker strukturiert worden: Bei der ersten Befragung 2004 gaben $46 \%$ der befragten Ärzte an, in ihrem Krankenhaus existierten „klare und koordinierte Abläufe von der Aufnahme bis zur Entlassung“, bei der zweiten Befragung 2006 waren es schon fast $60 \%$. Auch Abteilungen für Kurzzeitpatienten und poststationäre Versorgung gibt es 2006 häufiger als 2004 .

Der weitaus größte Teil der Patienten ist zu beiden Befragungszeitpunkten mit den Abläufen zufrieden: Jeweils knapp $90 \%$ gaben an, dass Untersuchungen und Behandlungen wie geplant durchgeführt worden sind. Verschlechterungen bei den Abläufen gibt es aus Patientensicht bei Aufenthalten in privaten Krankenhäusern: Hier hat sich der Anteil der Patienten, bei denen immer alles wie geplant ablief, zwischen den Befragungszeitpunkten von einem allerdings überdurchschnittlichen Niveau ausgehend um 8 Prozentpunkte verringert.

Eine weitere Teilpopulation der Patienten, die eine deutliche Verschlechterung der Versorgung wahrgenommen hat, sind die multimorbiden Patienten. Sie fühlten sich 2005 deutlich seltener vorbildlich betreut und mit Aufmerksamkeit behandelt. Der Rückgang der empfundenen Qualität beträgt in der Frage nach der vorbildlichen Betreuung 7 Prozentpunkte und in der Frage nach der aufmerksamen Behandlung ca. 12 Prozentpunkte. Bei den Patienten, die nur einen Aufnahmegrund angaben, gab es in diesen Fragen nur Veränderungen um ca. 1 Prozentpunkt oder weniger.
Graphik 5: Anteil der Patienten mit "immer" wie geplant durchgeführten Untersuchungen und Behandlungen nach Trägerschaft des Krankenhauses, 2002 und 2005

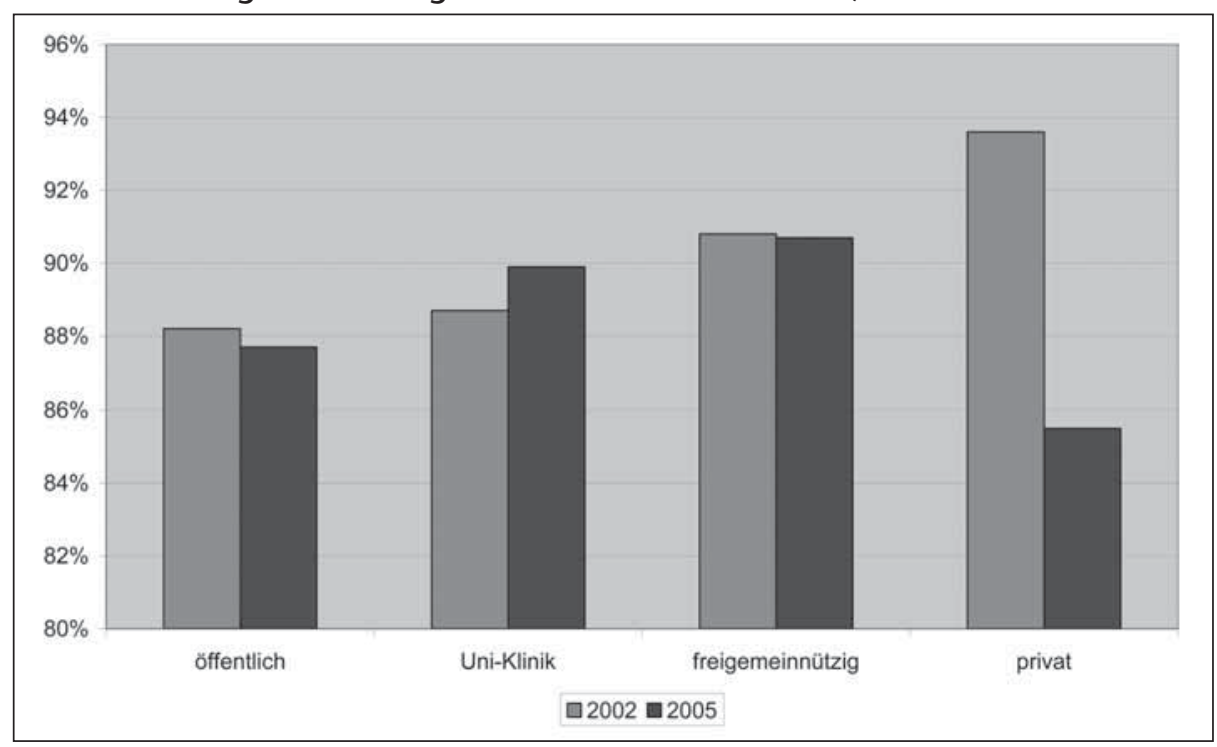

Quelle: Zusammenstellung aus Ergebnissen des Projektes WAMP 


\section{Reibungsverluste bei der Kooperation zwischen Berufsgruppen?}

In einem arbeitsteiligen System wie dem Krankenhaus kommt der Zusammenarbeit der verschiedenen Akteure grundsätzlich große Bedeutung zu. Im Zeitalter der DRGs wird die Kooperation zwischen Ärzten und Pflegekräften wichtiger denn je. Wenn Patienten in immer kürzerer Zeit behandelt werden sollen, muss sichergestellt sein, dass die Handlungen der verschiedenen Prozessbeteiligten aufeinander abgestimmt sind und die Kommunikation funktioniert. Sonst sind Verzögerungen, aber auch Fehler vorprogrammiert, die die Genesung behindern und somit zu Lasten der Patienten und des Krankenhauses gehen können. „Das Überwinden einer berufsgruppenorientierten Perspektive, zugunsten der patientenorientierten Sichtweise und optimierten interdisziplinären Steuerung der Behandlungs- und Versorgungsprozesse ist eine der zentralen Überlebensstrategien der Krankenhäuser im G-DRG-Zeitalter. ... Die steigende Arbeitsbelastung im ärztlichen Dienst, bedingt durch die Reduktion der Verweildauer und den erhöhten Dokumentationsaufwand, wird zur Folge haben, dass der Arzt noch weniger Zeit für die Arbeit auf der Station hat und mit dem Kunden verbringen kann. Somit kommt dem zeitnahen, interdisziplinären Informationsaustausch über den Behandlungsverlauf und möglicher Zusatzerkrankungen noch mehr Bedeutung zu“ (Wieteck 2005: 116, 119).

Ob sich die Kooperation zwischen Ärzten und Pflegekräften im Zusammenhang mit der Einführung der DRGs verbessert hat, lässt sich aufgrund der bisher vorliegenden Ergebnisse noch nicht abschließend sagen. In unseren qualitativen Fallstudien gibt es einerseits Hinweise auf vorhandene Kommunikations- und Kooperationsdefizite: z. B. nehmen Pflegekräfte in einigen Abteilungen aus Zeitgründen nicht mehr an der Visite teil. ${ }^{7}$ Andererseits gibt es Indizien für eine Wiederannäherung über die professionellen Grenzen hinweg, nachdem es im Zuge der Professionalisierung der Pflege einige Zeit deutliche Abgrenzungstendenzen gegeben hatte. Es werden integrierende, prozessorientierte Konzepte gefordert und zum Teil bereits umgesetzt, die auch eine Aufweichung der strikten Aufgabenteilung zwischen den Berufsgruppen beinhalten. Die Annäherung hängt dabei nicht zuletzt auch damit zusammen, dass die Pflegekräfte aus Angst um den Arbeitsplatz eher als früher zu Zugeständnissen bereit sind, etwa was die Übernahme ärztlicher Tätigkeiten angeht.

„Ich denke, dass das durch die DRGs eher besser wird. Weil alle sehen, weil alle viel mehr sehen, dass die wirtschaftlichen Zwänge für uns alle von ganz großer Bedeutung sind. Wenn wir nicht alle an einem Strang ziehen, dann geht es mit allen bergab. (...) Der Bettenleerstand ist höher als früher. Das bedeutet auch, dass der Bedarf an Pflegepersonal eigentlich sinkt. Das ist denen auch bewusst. Das bedeutet, wenn sie ihren Stand halten wollen, personell, so wie er jetzt ist, müssen sie langfristig andere Aufgabenbereiche übernehmen. Oder aber, wenn sie weiter diese strikte Mauerpolitik fahren wie bisher, dann wird die Anzahl der Pflegekräfte langfristig sinken. Es ist gar keine Frage, weil die Bettenzahl sinkt. Das ist denen auch bewusst. Deshalb sind die auch kompromissbereiter als früher. Deshalb denke ich, dass die DRGs in dieser Hinsicht für die Zusammenarbeit zwischen Pflege und Ärzteschaft günstig sind." (Chefarzt, B103, 216)

Insoweit lassen die qualitativen Fallstudien also auf einen positiven Effekt der DRG-Einführung für die Kooperation zwischen den Berufsgruppen schließen. Dies bestätigt sich teilweise auch in den quantitativen Erhebungen:

Insgesamt beurteilen die weitaus meisten Ärzte und Pflegekräfte die Kooperation zwischen den beiden Berufsgruppen als gut oder sehr gut, wobei der Anteil bei den Ärzten zwischen 2004 und 2006 nochmals leicht von $86 \%$ auf $88 \%$ angestiegen ist. ${ }^{8}$ Sowohl Ärzte als auch Pflegekräfte wurden darüber hinaus explizit gefragt, wie sie den Einfluss der DRGs auf verschiedene Aspekte des Arbeitslebens und des Versorgungsgeschehens beurteilen. „Zusammenarbeit" ist dabei ein Bereich, in dem beide Berufsgruppen vergleichsweise selten negative und vergleichsweise häufig positive Auswirkungen der DRGs wahrgenommen haben. So bewertete 2003 fast ein Fünftel der Pflegekräfte, die bereits unter DRG-Bedingungen arbeiteten, den Einfluss der DRGs auf die Kooperation mit Ärzten positiv. „Nur“ ein knappes Viertel sah zu dem Zeitpunkt negative Auswirkungen. Zum Vergleich: Negative Auswirkungen auf die allgemeinen Arbeitsbedingungen und die Zufriedenheit wurden von fast der Hälfte der Pflegekräfte beschrieben. Auch die Ärzte nehmen die Auswirkungen der DRGs auf die Kooperation mit den Pflegekräften mehrheitlich zumindest nicht negativ wahr. Allerdings ist der Anteil, der negative Auswirkungen auf die Kooperation mit Pflegekräften sieht, zwischen 2004 und 2006 leicht von 32\% auf 34\% angestiegen und der Anteil der positiven Stimmen von 7\% auf 5\% zurückgegangen. Der Rest sieht keinen Einfluss der DRGs oder traut sich kein Urteil zu.

\section{Fazit und sozialpolitische Schlussfolgerungen}

In Deutschland gibt es bisher keine offizielle DRGFolgen-Forschung. Der mögliche und sicherlich notwendige "Lernprozess" kann sich daher fast nur auf situative und möglicherweise tendenziöse und zufällige Wahrnehmungen stützen. Insofern sind die hier auszugsweise vorgestellten Ergebnisse des Projektes WAMP von großer Bedeutung, wenn es darum geht, festzustellen, welche erwünschten und nicht erwünschten Wirkungen die DRG-Einführung für die Versorgungsqualität hat bzw. an welchen Stellen Verbesserungen der Strukturen und Prozesse im Krankenhaus vorgenommen werden müssen, um negative Folgen der DRGs für die Patienten und Beschäftigten zu verhindern.

Die Erhebungen des Projekts WAMP haben zu einer Reihe von Ergebnissen geführt, die auf den ersten Blick nicht dramatisch erscheinen bzw. keine oder sogar 
positive Veränderungen in der bisherigen Zeit der DRGEinführung erkennen lassen. Dieses kann zum einen das Resultat nur sehr langsamer Anpassungsprozesse, zum anderen sich in der Summe aufhebender, im Detail völlig entgegengesetzt verlaufender Entwicklungen sein.

So können die Befunde von WAMP auf der einen Seite dazu beitragen, die Diskussion über die DRGs zu versachlichen oder auch zu entdramatisieren:

Im Vorfeld der DRG-Einführung wurden zum Teil extreme Auswirkungen für die Patientenversorgung befürchtet. Wie die Ergebnisse von WAMP zeigen, sind diese Folgen, z. B. die so genannten „blutigen“ Entlassungen, zumindest bisher nicht in großem Ausmaß aufgetreten.

Die ärztlichen und pflegerischen Handlungsorientierungen erweisen sich auch unter veränderten Rahmenbedingungen als relativ stabil, so dass weiterhin Spielräume zu Gunsten der Patienten genutzt werden (vgl. dazu auch Vogd 2006).

Angesichts der erforderlichen Liegezeitverkürzungen kommt es zu strukturierteren Behandlungsabläufen. Dies ist möglicherweise mit ein Grund dafür, dass die Zufriedenheit der Patienten mit der Behandlung insgesamt relativ konstant bleibt.

Entsprechend der veränderten Anforderungen durch die kürzeren Liegezeiten und den Dokumentationsaufwand kommt es auch zu einer Neujustierung der Arbeitsteilung zwischen den Ärzten und der Pflege. Insgesamt hat sich die Kooperation dieser Berufsgruppen nicht verschlechtert.

Auf der anderen Seite verweisen die Ergebnisse von WAMP aber auch auf verschiedene Problemaspekte, die bei der weiteren Umsetzung des DRG-Systems besonders beachtet werden müssen:

An den externen Schnittstellen im Krankenhaus gibt es Optimierungspotenzial. Sowohl bei der Aufnahme als auch bei der Entlassung treten für die Ärzte und für die Patienten Informationsdefizite auf. Dies kann nachteilig für den Patienten und auch für das Krankenhaus sein. Einerseits ist durch den mangelhaften Informationsfluss die Versorgung der Patienten nicht optimal und andererseits verteuert sich für das Krankenhaus der Krankenhausfall, wenn er auf Grund suboptimaler Informationslage länger dauert oder der Patient erneut aufgenommen werden muss. Der Integration der verschiedenen Versorgungsbereiche muss also in Zukunft noch stärkere Aufmerksamkeit gewidmet werden.

Schaut man sich die Entwicklung der Krankenhäuser danach an, welcher Trägerform sie angehören, fallen in mancher Hinsicht die privaten Krankenhäuser besonders auf. Bei einzelnen Indikatoren treten aus Sicht ihrer Patienten nur in ihnen teilweise deutliche Verschlechterungen auf.

In den quantitativen und qualitativen Erhebungen gibt es Hinweise darauf, dass vor allem chronisch kranke und multimorbide Patienten ${ }^{9}$ durch die Neuregelungen im DRG-System benachteiligt werden. Hier muss gegengesteuert werden, um auch in Zukunft die soziale Qualität der Versorgung, also etwa die Chancengleichheit beim Erhalt von Gesundheitsleistungen zu gewährleisten.

In diesem Zusammenhang muss insbesondere vor einem weiteren Personalabbau im Bereich der Pflege gewarnt werden, da dieser vor allem zu Lasten der psychosozialen Versorgung und aktivierender Maßnahmen geht.

\section{Literatur}

Bähr-Heintze, Karin, 2004: "Wer nur an die Kosten denkt, denkt zu kurz", Pflegezeitschrift (10): 698-701.

Böcking, Wolfgang; Ahrens, Ulrich; Kirch, Wilhelm; Milakovic, Mishael, 2005: "First results of the introduction of DRGs in Germany and overview of experience from other DRG countries", Public Health 13 128-137.

Braun, Bernard; Müller, Rolf, 2003: Auswirkungen von Vergütungsformen auf die Qualität der stationären Versorgung. St. Augustin: Asgard.

Braun, Bernard; Müller, Rolf, 2006: Versorgungsqualität im Krankenhaus aus der Perspektive der Patienten. St. Augustin: Asgard.

Braun, Bernard; Müller, Rolf; Timm, Andreas, 2004: Gesundheitliche Belastungen, Arbeitsbedingungen und Erwerbsbiografien von Pflegekräften im Krankenhaus. Eine Untersuchung vor dem Hintergrund der DRG-Einführung. St. Augustin: Asgard.

Buhr, Petra; Klinke, Sebastian, 2006: Versorgungsqualität im DRGZeitalter. Erste Ergebnisse einer qualitativen Studie in vier Krankenhäusern. Bremen: Zentrum für Sozialpolitik (6/2006),

Deutscher Bundestag, 2001: Gesetzentwurf der Fraktionen SPD und BÜNDNIS 90/ die GRÜNEN. Entwurf eines Gesetzes zur Einführung des diagnose-orientierten Fallpauschalensystems für Krankenhäuser (Fallpauschalengesetz - FPG). Drucksache 14/6893. Berlin: Deutscher Bundestag (14/6893),

Dietrich, Frank; Imhoff, Michael; Kliemt, Hartmut, 2004: Standardisierung in der Medizin. Qualitätssicherung oder Rationierung? Stuttgart, New York: Schattauer.

Friedrich, Jörg; Günster, Christian (2006): Determinanten der Casemixentwicklung in Deutschland während der Einführung von DRGs (2002 bis 2004). In: Klauber, Jürgen; Robra, Bernt-Peter, Schellschmidt, Henner (Hrsg.): Krankenhaus-Report 2005. Schwerpunkt: Wege zur Integration. Stuttgart: Schattauer.

Hausner, Elke; Juchems, Stefan; Richter, Inga; Schulze Geiping, Annika; Simon, Mario; Voß, Karin; Wiedemann, Regina; Donath, Elke; Bartholomeyczik, Sabine, 2005: "Arbeitsstrukturen in der Pflege im Krankenhaus und die Einführung der DRG", Pflege \& Gesellschaft 10 (3): $125-130$.

InEK, Institut für das Entgeltsystem im Krankenhaus, 2006: Datenauswertung §21 KHEntgG zur Begleitforschung gemäß §17b Abs. 8 KHG; Datenjahr 2004 (Stand 14.03.2006). Siegburg: Institut für das Entgeltsystem im Krankenhaus www.inek-drg.de

Klinke, Sebastian; Kühn, Hagen, 2006: Krankenhaus, Geld und ärztliche Arbeit. Eine sozialwissenschaftliche Studie zur finanziellen Steuerung der Krankenhäuser. Berlin: Edition Sigma.

Lauterbach, Karl W.; Lüngen, Markus, 2000: DRG-Fallpauschalen: eine Einführung. Stuttgart, New York: Schattauer.

Leister, Jan Eric; Stausberg, Jürgen, 2005: "Comparison of cost accounting methods from different DRG systems and their effect on health care quality", Health Policy (74): $46-55$.

Lenk, Christian; Biller-Andorno, Nikola ; Alt-Epping, Bernd; Anders, M.; Wiesemann, Claudia, 2005: "Ethik und Fallpauschalen. Welche Veränderungen in der Patientenversorgung sind zu erwarten?" Deutsche Medizinische Wochenschrift 130 1653-1655.

Loch, Markus, 2005: "Fast Track oder der schnellstmögliche Behandlungsweg", Pflege Aktuell (2): 91-93.

Oberender, Peter O., 2005: Clinical Pathways. Facetten eines neuen Versorgungsmodells. Stuttgart: Kohlhammer.

Raffel, Andreas; Cupisti, Kenko; Dotzenrath, Cornelia; Krüger, B.; Ohmann, Christian; Schulte, Klaus-Martin; Goretzki, Peter Erich; Röher, Hans-Dietrich, 2004: "Ökonomische Zwänge führen zur Reduktion der stationären Verweildauer. Beispiel: Schilddrüsenoperation", Der Chirurg 75 702-705.

Roeder, Norbert, 2005: Anpassungsbedarf der Vergütung von Krankenhausleistungen für 2006. Münster: DRG-Research-Group, Universitätsklinikum Münster Westfälische Wilhelms-Universität 
Rühmkorf, Daniel, 2005: "Heilen im Minutentakt", Dr. med. Mabuse 153 $25-27$.

Simon, Michael, 2000: "Was die Umstellung auf DRG für die Krankenhäuser bedeutet", Pflegezeitschrift (11): 734-737.

Statistisches Bundesamt, 2006: Fachserie 12, Reihe 6.1.1, Gesundheitswesen. Grunddaten der Krankenhäuser, 2004. Wiesbaden:

Vogd, Werner, 2004: Ärztliche Entscheidungsprozesse des Krankenhauses im Spannungsfeld von System- und Zweckrationalität. Berlin: VWF Verlag.

Vogd, Werner, 2006: Die Organisation Krankenhaus im Wandel. Bern: Huber.

von Eiff, Wilfried; Klemann, Ansgar; Middendorf, Conrad, 2005: REDIAStudie. Analyse der Auswirkungen der DRG-Einführung auf die medizinische Rehabilitation. Münster: LIT Verlag.

Wieteck, Pia, 2005: "Zur Bedeutung der interdisziplinären Zusammenarbeit im Kontext der DRG", Pflege \& Gesellschaft 10 (3): 115 - 124.

Wilmsen-Neumann, Jürgen, 2005: "Stirbt die ganzheitliche Sicht des Menschen? Das deutsche DRG-System und die Palliativmedizin", Klinikarzt 34 24-28.

Wingenfeld, Klaus, 2005: Die Entlassung aus dem Krankenhaus: Institutionelle Übergänge und gesundheitlich bedingte Transitionen. Bern: Huber.

\section{Fußnoten}

1 Vgl. z. B. Böcking et al. (2005); Lauterbach/Lüngen (2000); Leister/Stausberg (2005); Lenk et al. (2005); Raffel et al. (2004); Rühmkorf (2005); Simon (2000); Wilmsen-Neumann (2005).

2 Das DRG-Institut InEK hat im März 2006 eine erste tabellarische Auswertung von Strukturdaten (z. B. Fallzahl, Verweildauer, CMI) aufgrund der Datenlieferungen der Krankenhäuser gemäß § 21 Krankenhausentgeltgesetz vorgelegt (InEK 2006). An der Universität Münster beschäftigt sich die DRG Research Group mit der Evaluation von DRG-Systemen, wobei es vor allem um die Abbildungsgenauigkeit des Systems, die Analyse von Schwachstellen und Vorschläge zur Weiterentwicklung des Systems geht (www.drg-research.de sowie Roeder 2005). Die Bundesgeschäftsstelle Qualitätssicherung hat ein System von Indikatoren zur Abbildung der medizinischen Qualität entwickelt und Qualitätsreports veröffentlicht (weitere Informationen unter www.bqs-online.de). Schließlich gibt es eine Reihe von größeren oder kleineren Forschungsprojekten. Zu erwähnen sind hier insbesondere die REDIA (Rehabilitation und Diagnosis Related Groups) - Studie am Institut für Krankenhausmanagement der Universität Münster (von Eiff et al. 2005), die sich mit den Folgen der DRG für die Rehabilitation beschäftigt, die Untersuchungen von Werner Vogd (Freie Universität Berlin) zu ärztlichen Entscheidungsprozessen (Vogd 2004, 2006), eine Longitudinalstudie zu den Auswirkungen der DRG auf die Pflege an der Universität Witten-Herdecke (Hausner et al. 2005) sowie das am Institut für Medizinmanagement und Gesundheitsökonomie der Universität Bayreuth angesiedelte Projekt zu den Auswirkungen der DRG auf kirchliche Krankenhäuser.

3 Das Projekt wird gefördert bzw. unterstützt von der Hans-BöcklerStiftung, der Gmünder Ersatzkasse (GEK), der Gewerkschaft Ver.di und der Landesärztekammer Hessen. Es wird geleitet von Bernard Braun und Hagen Kühn. Mitarbeiter sind Petra Buhr, Sebastian Klinke und Rolf Müller.

$4 \mathrm{Zu}$ den bisherigen Ergebnissen des Projekts siehe Braun/Müller (2003, 2006); Braun et al. (2004); Klinke/Kühn (2006); Buhr/Klinke (2006).

5 So wichtig diese Art von Ausgangs- oder "Nullpunkt“-Wissen ist, so schwierig ist die Identifikation eines Nullpunkts und das Auseinanderhalten von DRG-Auswirkungen, Auswirkungen der Antizipation der Einführung und Auswirkungen, die nichts mit den DRGs zu tun haben, sondern beispielsweise der Kostendämpfungspolitik von GKV und öffentlichen Händen oder der traditionellen KrankenhausHierarchie geschuldet sind.

6 Die sog. Konvergenzphase wurde durch das zweite Fallpauschalenänderungsgesetz um zwei Jahre bis Ende 2008 verlängert. Wir gehen dabei davon aus, dass die Krankenhäuser nicht erst am Ende der Konvergenzphase reagieren, sondern sich bereits während der Konvergenzphase durch gezielte Veränderungen auf die neue Situation einstellen. Die Anpassungsquote der individuellen Krankenhausfallwerte an den landeseinheitlichen Fallwert steigt von 15 Prozent 2005 auf 20 Prozent 2006 und 2007. Zugleich steigt die so genannte Kappungsgrenze, die festlegt, um wie viel ein Krankenhausbudget durch die Anpassung maximal gesenkt werden darf, von 1 Prozent auf 2 Prozent.

7 Wie unsere quantitativen Erhebungen zeigen, ist die Visite nach Aussage der Ärzte nach wie vor der wichtigste Ort, an dem Informationen zwischen Ärzten und Pflegekräften ausgetauscht werden. Bei der zweiten Befragung 2006 ist der Anteil der Ärzte, die angeben, dass Pflegekräfte meistens oder häufig in der Visite konsultiert werden, allerdings leicht von $75 \%$ auf $72 \%$ gesunken. Die Häufigkeit von Besprechungen mit Pflegekräften hat sich zwischen den beiden Befragungswellen dagegen leicht erhöht.

8 Bei den Pflegekräften beurteilten 2003 drei Viertel die Zusammenarbeit mit den Ärzten als sehr gut oder eher gut. Dabei gab es keine signifikanten Unterschiede zwischen Pflegekräften aus Optionshäusern und Krankenhäusern, die noch keine DRG eingeführt hatten.

Ergebnisse der zweiten Pflegekräftebefragung liegen noch nicht vor.

9 Dieser Aspekt wurde in diesem Beitrag nur ansatzweise behandelt. Vgl. dazu ausführlich Braun/Müller (2006: 83 ff.)

\title{
Humane Selbstbestimmung
}

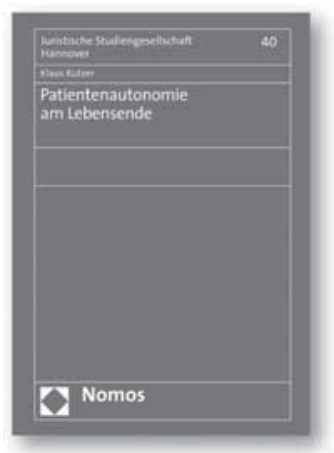

\author{
Patientenautonomie am Lebensende \\ Von Klaus Kutzer \\ 2006, 44 S., Rückendrahtheftung, 8,- €, ISBN 3-8329-2095-1 \\ (Juristische Studiengesellschaft Hannover, Bd. 40)
}

Die rechtlichen und ethischen Aspekte der Sterbebegleitung und die verschiedenen Formen der Sterbehilfe sind zurzeit Gegenstand umfangreicher und streitiger Erörterung in den verschiedensten Gremien.

Der Band gibt einen Überblick über die aktuelle Diskussion in Fragen der aktiven und passiven Sterbehilfe, der Reichweite und Form einer Patientenverfügung, der Einschaltung des Vormundschaftsgerichts, der indirekten Sterbehilfe und des ärztlich assistierten Suizids.

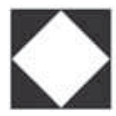

\section{Nomos}

Bitte bestellen Sie bei Ihrer Buchhandlung oder bei: Nomos Verlagsgesellschaft | 76520 Baden-Baden | www.nomos.de 